\title{
From prevention to optimal treatment in chronic rhinosinusitis
}

Prevention of chronic rhinosinusitis remains a true societal challenge, as prevention of this highly prevalent non-communicable disease will significantly reduce socio-economic costs and improve the overall well-being of the large group of affected individuals. In alignment with the health priorities of national governments, prevention and cost-effective health care should be embraced by the Rhinologic community. In the 2018 December issue of Rhinology, Hopkins et al. ${ }^{(1)}$ clearly identify key areas of action for primary, secondary and tertiary prevention, allowing a structured action plan for the near future. The need for action is illustrated by Hoven and coworkers ${ }^{(2)}$ highlighting the correlation between self-reported sino-nasal dysfunction and daytime sleepiness. Interestingly, associations were mainly found between symptoms of mucosal inflammation, like nasal discharge, headache, coughing and sneezing, rather than nasal obstruction. In addition, the impact of chronic rhinosinusitis exacerbations on work productivity and absenteïsm is confirmed by the research work by Philips ${ }^{(3)}$. Besides prevention of chronic rhinosinusitis, also reduction of the complication risk of endoscopic sinus surgery is of paramount importance. Preti et al. ${ }^{(4)}$ analyzed the risk of iatrogenic cerebrospinal fluid leak in relation to the Keros or Gera classification. The Gera classification turned out to be more sensitive to anatomical variations associated with cerebrospinal fluid leaks. In addition, Mak et al. (5) report on the value of staging systems predicting recurrent inverted papilloma, with the Han and Cannady staging being more valuable than other staging systems.

Promising novel surgical options emerge for patients with nasal valve dysfunction and medium-sized septal perforations. In a series of 32 patients ${ }^{(6)}$, the butterfly titanium implant showed significant improvement of breathing, even with 28 out of 32 patients having had previous nasal surgery. Santamaria-Gadea et al. ${ }^{(7)}$ propose a modified simple and extended nasal floor and inferior meatus flap for the reconstruction of small and medium-sized septal perforations. Regardless of tumor stage, squamous cell carcinoma of the nasal vestibule can be treated primarily with surgery ${ }^{(8)}$, with adjuvant radiotherapy only needed for advanced stage cancer.

Novel insight into therapeutic options is mandatory to better help those suffering from sinonasal disease. In patients with post-traumatic or post-infectious olfactory loss, olfactory training represents an effective treatment, with Hummel et al. ${ }^{(9)}$ reporting for the first time the associated increase in electroolfactogram responses to pleasant and unpleasant odors. In an attempt to further explore novel therapeutic pathways for anosmia, Li et al. ${ }^{(10)}$ utilized olfactory neuroepithelial cells from embryonic rats to assess the effects of chitosan on neurosphere formation and differentiation of olfactory receptor neurons, with promising findings. Smell disorders in relation to drug intake in the elderly, has been studied by Ottaviano et al. (11), confirming the clinical observation of correlation between the number of drugs taken for non-rhinologic conditions and smell impairment. Diving deeper into immune mechanisms of CRSwNP, Wang ${ }^{(12)}$ reports on the regulation of eotaxin-1 production by Thymic Stromal Lymphopoetin (TSLP), linking epithelial immune activation to eosinophilic influx. Better insight in different inflammatory profiles found in CRSwNP and antrochoanal polyps is provided by Jin and coworkers ${ }^{(13)}$.

Overlooking the excellent research work by different groups world-wide published in the latest issue of Rhinology, all members of the European Rhinologic Society should be very excited about the promising and dynamic field of Rhinology!

\section{References}

1. Hopkins C, Surda P, Bast F, Hettige R, Walker A, Hellings PW. Prevention of chronic rhinosinusitis. Rhinology. 2018; 56, 4: 307-315.

2. Hoven KM, Aarstad HJ, Bjorvatn B, Lundemo EH, Steinsvåg SK. Correlation between Excessive Daytime Sleepiness (EDS) and self- reported and objective nasal characteristics. Rhinology. 2018; 56, 4: 316-322.

3. Phillips KM, Bergmark RW, Hoehle LP, et al Chronic rhinosinusitis exacerbations are differentially associated with lost productivity based on asthma status. Rhinology. 2018; 56, 4: 323-329.

4. Preti A, Mozzanica F, Gera R, et al. Horizontal lateral lamella as a risk factor for iatrogenic cerebrospinal fluid leak. Clinical retrospective evaluation of 24 cases. Rhinology. 2018; 56, 4: 358-363.

5. Mak W, Webb D, Al-Salihi S, Dadgostar A, Javer A. Sinonasal inverted papilloma recurrence rates and evaluation of current staging systems. Rhinology. 2018; 56, 4: 407-414.

6. van den Broek SJAC, van Heerbeek N. The effect of the titanium butterfly implant on nasal patency and quality of life. Rhinology. 2018; 56, 4: 364-369.

7. Santamaría-Gadea A, López-Chacón M Langdon C, et al. Modified nasal floor and inferior meatus flap for septal perforation repair. Extension and limits. Rhinology. 2018; 56, 4: 386-392.

8. Zaoui K, Plinkert PK, Federspil PA. Primary surgical treatment of nasal vestibule cancer - therapeutic outcome and reconstructive strategies. Rhinology. 2018; 56, 4: 393-399. 
9. Hummel T, Stupka G, Haehner A, Poletti SC Olfactory training changes electrophysiological responses at the level of the olfactory epithelium. Rhinology. 2018; 56, 4: 330335.

10. Li ST, Young TH, Wang CT, Huang TW. Chitosan films promote formation of olfactory neurospheres and differentiation of olfactory receptor neurons. Rhinology. 2018; 56, 4: 336-342.

11. Ottaviano G, Savietto E, Scarpa B, et al. Influence of number of drugs on olfaction in the elderly. Rhinology. 2018; 56, 4: 351357.

12. Wang WW, Lu DM, Zheng M, Zhang JG, Zhang B. TSLP regulates eotaxin-1 production by nasal epithelial cells from patients with eosinophilic CRSwNP. Rhinology. 2018 56, 4: 370-377.

13. Jin P, Zi X, Charn TC, et al. Histopathological features of antrochoanal polyps in Chinese patients. Rhinology. 2018; 56, 4: 378-385.

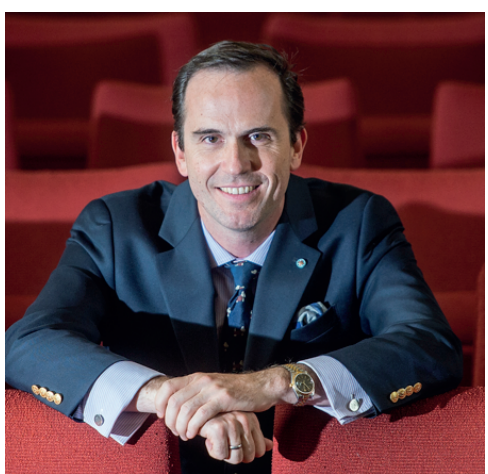

Peter W. Hellings, Associate Editor Leuven, Belgium 